\title{
Variabilidade média do perfil da temperatura e da umidade relativa do ar em uma floresta tropical chuvosa na Amazônia brasileira
}

\author{
Average variability of temperature and relative humidity of the air in a tropical rain \\ forest in the Brazilian Amazon
}

\author{
Antônio Carlos Lôla da Costa', João de Athaydes Silva Junior', Alex Antônio Ribeiro de Oliveira"l, \\ Lucy Rowland ${ }^{\mathrm{III}}$, Patrick Meirlv, Hernani José Brazão Rodrigues' ${ }^{1}$, Carlos Leandro Ribeiro da Costal \\ Universidade Federal do Pará. Belém, Pará, Brasil \\ "Museu Paraense Emílio Goeldi/MCTIC. Belém, Pará, Brasil \\ I'University of Exeter. Exeter, Inglaterra \\ vUniversity of Edinburgh. Edinburgh, Escócia
}

\begin{abstract}
Resumo: A floresta amazônica apresenta elevadas temperaturas e grandes quantidades de precipitações anuais, embora ocorram grandes variações interanuais desses elementos meteorológicos. A temperatura do ar (Tar) e a umidade relativa do ar (UR) dentro e acima de uma floresta são resultado de complexas trocas energéticas através dos processos de reflexão, transmissão e absorção da energia solar. Esse estudo foi realizado na Floresta Nacional de Caxiuanã, Pará, Brasil, e teve como objetivo principal avaliar a variabilidade sazonal da temperatura e umidade do ar a partir de uma análise de perfil vertical no interior da floresta, com medidas alturas de $2 \mathrm{~m}, 16 \mathrm{~m}, 28 \mathrm{~m}$ e $42 \mathrm{~m}$, com registros a cada 30 minutos, durante o período de 2012 a 2016. Os resultados indicaram grande sazonalidade nestes elementos meteorológicos, e as maiores temperaturas ocorreram no nível do dossel (28 m), e as menores foram observadas próximo à superfície (2 m), devido a atenuação da radiação solar no interior da floresta. Os maiores valores da UR foram observados próximo à superfície (2 m), e os menores ocorreram acima do dossel, devido as maiores velocidades do vento neste nível. Estes resultados indicam grande variabilidade espaço-temporal destes elementos meteorológicos, o que influencia no comportamento dos organismos vivos que habitam aquele ambiente florestal.
\end{abstract}

Palavras-chave: Floresta tropical. Sazonalidade. Elementos meteorológicos.

\begin{abstract}
The Amazon rainforest presents high temperatures and annual precipitation, although there are large interannual variations in these meteorological elements. Air temperature (Tar) and relative air humidity $(\mathrm{RH})$ in and above a forest are the result of complex energy exchanges through the processes of reflection, transmission, and absorption of solar energy. This study was carried out in the Caxiuanã National Forest, Pará, Brasil, and the objective was to evaluate the seasonal variability of air temperature and humidity from a vertical profile analysis within the forest with measures heights of 2,16 , 28 and $42 \mathrm{~m}$, with readings taken every 30 minutes from 2012 to 2016 . The results indicated a great seasonality in these meteorological elements, since the highest temperatures occurred at the canopy level $(28 \mathrm{~m})$, and the lowest ones were observed near the surface $(2 \mathrm{~m})$ due to the attenuation of solar radiation inside the forest. The highest $\mathrm{RH}$ values were observed near the surface $(2 \mathrm{~m})$, and the lowest values occurred above the canopy, due to the higher wind speeds at this level. These results indicate a large spatial-temporal variability of these meteorological elements, which influence the behavior of living organisms that inhabit that forest environment.
\end{abstract}

Keywords: Tropical forest. Seasonality. Meteorological elements.

COSTA, A. C. L., J. A. SILVA JUNIOR, A. A. R. OLIVEIRA, L. ROWLAND, P. MEIR, H. J. B. RODRIGUES \& C. L. R. COSTA, 2018. Variabilidade média do perfil da temperatura e da umidade relativa do ar em uma floresta tropical chuvosa na Amazônia brasileira. Boletim do Museu Paraense Emílio Goeldi. Ciências Naturais 13(2): 261-269.

Autor para correspondência: Antônio Carlos Lôla da Costa. Universidade Federal do Pará. Instituto de Geociências. Faculdade de Meteorologia. Rua Augusto Corrêa, 1 - Guamá. Belém, PA, Brasil. CEP 66075-110 (lola@ufpa.br).

Recebido em 07/08/2017

Aprovado em 02/09/2017

Responsabilidade editorial: Fernando da Silva Carvalho Filho

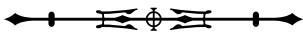




\section{INTRODUÇÃO}

A floresta amazônica possui área aproximada de 5,5 x $10^{6} \mathrm{~km}^{2}$, com pluviosidade média anual de $2.300 \mathrm{~mm}$. $\bigcirc$ seu principal corpo hídrico, o rio Amazonas, efetua uma descarga líquida média anual de $200.000 \mathrm{~m}^{3} \cdot \mathrm{s}^{-1}$ no oceano Atlântico (Malhi et al., 1998). A quantidade de pesquisas realizadas na região vem crescendo nas últimas décadas devido às características peculiares de fauna e de flora do local, além do crescimento das pressões antrópicas, o qual vem causando impactos tanto no meio biótico como no físico (A. C. L. Costa et al., 2010).

O ecossistema amazônico exerce uma função muito importante no balanço hidrológico do planeta, em razão de cerca de 50\% da precipitação anual retornar para a atmosfera por meio do processo de evapotranspiração (R. F. Costa et al., 2007). Em relação à precipitação, definem-se duas épocas distintas ao longo do ano, uma estação chuvosa, compreendida entre os meses de dezembro a maio, e outra menos chuvosa ou seca, que se estende de junho a novembro (A. C. L. Costa et al., 2004). Estas condições estão diretamente vinculadas à grande quantidade de radiação solar na região tropical, além da influência direta da zona de convergência intertropical (ZCIT), principal sistema meteorológico a atuar no regime de chuvas na região amazônica (Horel et al., 1989).

As florestas desempenham papel importante na regulação climática e atuam em todas as escalas, da local a global, pois modulam o comportamento dos fluxos de calor sensível e latente no ecossistema, exercendo relevante função no controle climático regional e globalmente (Malhi et al., 2008; Dantas et al., 2011).

A temperatura do ar é um dos elementos meteorológicos mais importantes e medidos, pois influencia diretamente os processos fisiológicos que ocorrem tanto na fauna quanto na flora, o que demonstra a importância do estudo dessa variável em ambientes florestais (Medeiros et al., 2005; WMO, 2008). Esta variável pode ser definida como uma quantidade física que caracteriza o movimento médio e aleatório das moléculas presentes no ar atmosférico, representando o estado termodinâmico da massa de ar que está sendo estudada.

A variação da temperatura do ar é essencial para o crescimento, o desenvolvimento e a sobrevivência dos indivíduos que habitam o interior das florestas, sendo este um elemento meteorológico de enorme importância, atuando diretamente na evapotranspiração e no balanço energético do local (A. C. L. Costa et al., 2004), sendo indispensável para a caracterização do microclima no interior das florestas.

A floresta amazônica possui um dos mais importantes ecossistemas da Terra, com a cobertura vegetal controlando grande parte da radiação solar incidente, a qual é absorvida pela superfície terrestre. Contudo, em razão do crescente desmatamento causado pela ação humana, este equilíbrio poderá ser interrompido, provocando grandes alterações no clima local, regional ou global.

A interação efetiva do clima e das estações de plantas foi bem estabelecida desde os primeiros estágios de pesquisa micrometeorológica (Geiger, 1942). Assim, o conhecimento dos processos de troca biofísica na interface vegetaçãoatmosfera, bem como a sua influência no microclima da floresta são muito relevantes na pesquisa ecológica, uma vez que o microclima afeta processos ecológicos, como regeneração e crescimento de plantas, respiração do solo, ciclagem de nutrientes e formação de habitat.

A estrutura do dossel de uma floresta apresenta especial interesse, pois seu microclima diferenciado atua diretamente em diferentes processos, como a fotossíntese, que é uma componente importante para o balanço de carbono da floresta (Funk \& Lerdau, 2004). No dossel das florestas tropicais, que varia de 20 a $40 \mathrm{~m}$ acima do solo, encontra-se grande parte da biodiversidade desse ambiente (Nicolasa et al., 2005).

A estrutura do dossel de uma floresta determina a quantidade de radiação solar que alcança o sub-bosque, assim como a quantidade de radiação solar que chega à superfície, onde a arquitetura dele influencia diretamente 
nos fluxos verticais de energia entre a atmosfera e o sub-dossel, sendo um dos sistemas biológicos mais complexos do planeta (Madigosky, 2004). Essa estrutura também influencia a quantidade de energia que é armazenada na biomassa. Portanto, para se conhecer melhor os impactos e o desenvolvimento dos ecossistemas florestais, faz-se necessário compreender e caracterizar os processos de variabilidade térmica que ocorrem no dossel florestal, pois estará relacionado diretamente à dinâmica da biodiversidade que habita o dossel florestal.

O presente estudo teve como objetivo principal determinar a variabilidade sazonal média do perfil de temperatura e de umidade relativa do ar em uma floresta tropical chuvosa na Amazônia brasileira, no sítio Experimental do Projeto Estudo da Seca na Floresta (ESECAFLOR), integrante do Programa de Grande Escala da Biosfera-Atmosfera na Amazônia (LBA), na Floresta Nacional (FLONA) de Caxiuanã, situada no estado do Pará.

\section{MATERIAIS E MÉTODOS}

\section{LOCAL DA PESQUISA}

A presente pesquisa foi desenvolvida na Estação Científica Ferreira Penna (ECFPn - 010 42' 30" S e 510 31' 45" W), localizada na FLONA de Caxiuanã, que é administrada pelo Museu Paraense Emílio Goeldi (MPEG), conforme pode ser visualizado na Figura 1. A ECFPn é uma base de pesquisa multidisciplinar onde atualmente se desenvolvem várias pesquisas, entre elas podemos citar: Projeto Rede de Avaliação e Monitoramento de Ecologia Tropical (TEAM), Programa de Pesquisas Ecológicas de Longa Duração (PELD) e Programa de Grande Escala da BiosferaAtmosfera na Amazônia (LBA).

No âmbito do programa LBA, o projeto ESECAFLOR, iniciado em 2001 com financiamento da comunidade europeia, e mantido até os dias atuais pelo Ministério da Ciência, Tecnologia, Inovações e Comunicações (MCTIC), tem como objetivo avaliar o impacto da redução da disponibilidade hídrica do solo sobre os fluxos de

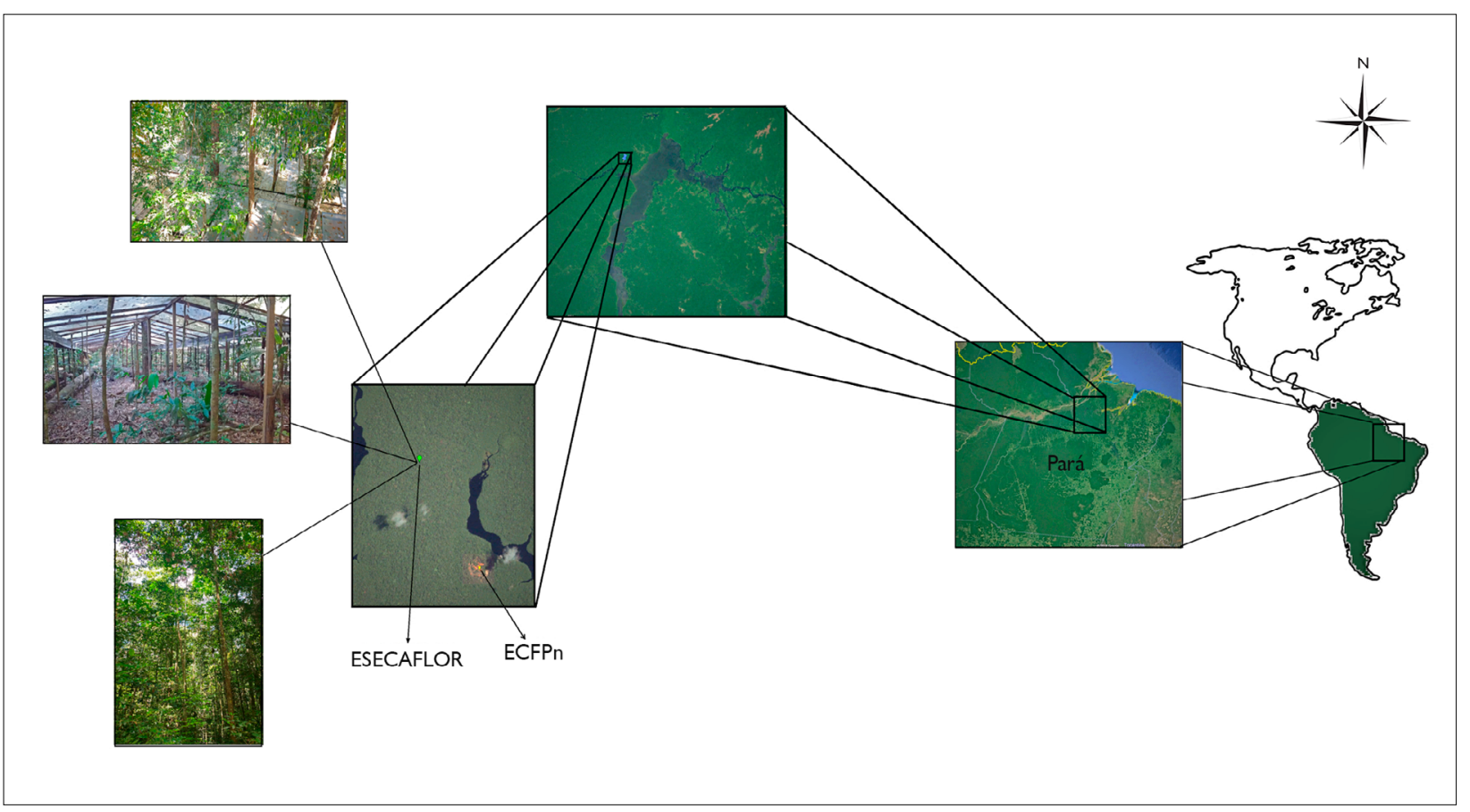

Figura 1. Localização da área de estudo na Floresta Nacional de Caxiuanã, Pará. Fotos: João de Athaydes Silva Junior. Mapa: Google Earth.

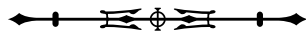


água e de dióxido de carbono em uma floresta tropical amazônica, algo semelhante à influência de um fenômeno El Niño. Em sua estrutura física, o projeto ESECAFLOR é composto por duas parcelas de um hectare cada. Estas áreas são delimitadas por trincheiras cavadas com profundidades variando de 50 a $150 \mathrm{~cm}$. A parcela A é usada como testemunha para os experimentos realizados na parcela $B$, onde está sendo feita a exclusão de, aproximadamente, 50\% da água da chuva. Esta exclusão foi realizada com a construção de uma estrutura composta por, aproximadamente, 6.000 painéis plásticos, medindo 0,50 × 3,0 m, distribuídos a uma altura variando de 1,5 a 3,5 m acima do solo. Toda a água captada é transportada através de calhas para uma trincheira, que a exclui por gravidade. Cada uma das áreas possui uma torre metálica com 40 m de altura. Estas torres permitem o acesso fácil às árvores para análises de fisiologia vegetal, entre outros experimentos, além do monitoramento realizado por estações meteorológicas automáticas.

A altura média dos indivíduos arbóreos deste ambiente florestal é de 40 m (Lisboa, 1997), com o solo do tipo Latossolo Amarelo (Ruivo et al., 2002). As áreas de estudo foram criteriosamente selecionadas, atentando para a composição basal e a predominância das espécies. Uma seca artificial do solo foi imposta na parcela de exclusão da chuva. Essa técnica já foi usada com sucesso na Floresta Nacional de Tapajós (Nepstad et al., 2002).

\section{DADOS UTILIZADOS}

No estudo do perfil vertical da temperatura e da umidade relativa do ar (Figura 2) foram utilizados equipamentos do tipo HMP45C (Temperature \& Relative Humidity Sensor),

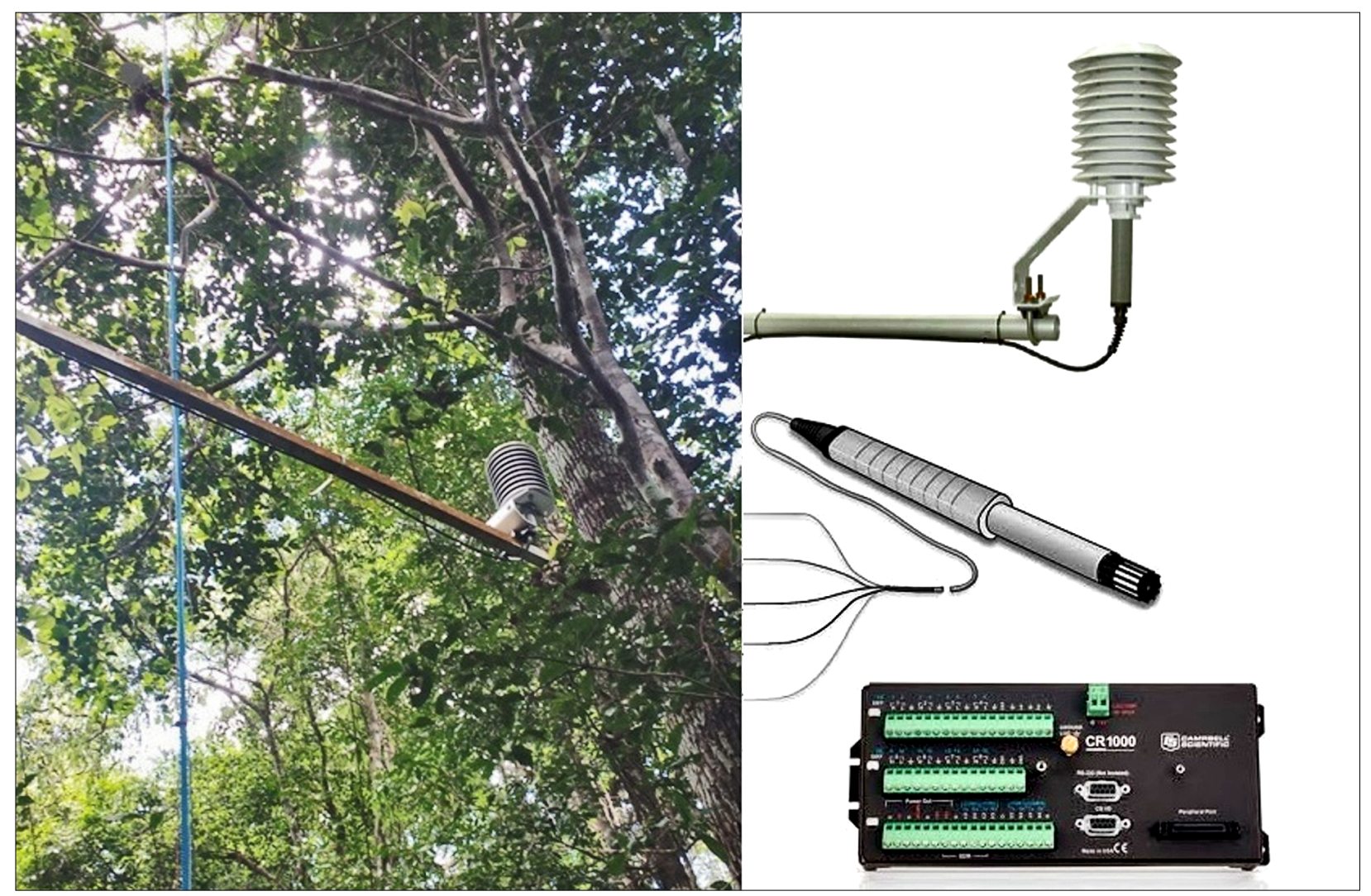

Figura 2. Sensor de temperatura e de umidade relativa do ar no abrigo e o datalogger para armazenamento dos dados. Foto: João de Athaydes Silva Junior. Desenhos: Campbell Scientific.

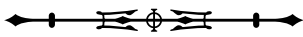


da Vaisala, instalados nas alturas de 2, 16, 28 e $42 \mathrm{~m}$ e conectados a um datalogger modelo CR1000, da Campbell Scientific. Os dados utilizados neste estudo referem-se ao período de 2012 a 2016, coletados em uma torre com $40 \mathrm{~m}$ de altura, situada na parcela de controle (floresta primária e conservada intacta) do projeto ESECAFLOR. Após a consistência dos dados, eles foram analisados na planilha eletrônica Excel.

\section{RESULTADOS E DISCUSSÕES}

\section{VARIABILIDADE MÉDIA ANUAL DO PERFIL DE TEMPERATURA DO AR}

Na Figura 3, temos a variabilidade média anual do perfil de temperatura do ar no sítio experimental do projeto ESECAFLOR/LBA, no período de 2012 a 2016. Ficou evidente a sazonalidade deste perfil, onde as menores temperaturas foram observadas justamente durante o período chuvoso da região, caracterizado por grande cobertura de nuvens e por chuvas abundantes, o que torna o ambiente menos aquecido em comparação com o período seco, onde estas características são grandemente alteradas. Observou-se que, de uma maneira geral, as menores temperaturas foram verificadas nos níveis mais próximos da superfície (2 e $16 \mathrm{~m}$, respectivamente). Neste caso, os valores médios oscilaram entre máximos de 25,9 ${ }^{\circ} \mathrm{C}$ a mínimos de $23,4^{\circ} \mathrm{C}$, com valores médios de $24,6^{\circ} \mathrm{C}$. Por outro lado, os maiores valores foram observados no nível de $42 \mathrm{~m}$, acima do dossel, com máximos e mínimos de $26,9^{\circ} \mathrm{Ce} 22,9^{\circ} \mathrm{C}$, respectivamente. O valor médio neste nível foi de $25,1^{\circ} \mathrm{C}$, portanto, $0,5^{\circ} \mathrm{C}$ maior do que o nível da superfície. O nível de 16 m foi onde se observou o segundo menor valor de temperatura deste perfil, pois ainda encontra-se abaixo do dossel vegetativo, tendo a média apresentado um valor de $25,0^{\circ} \mathrm{C}$, com máximos e mínimos de $26,6^{\circ} \mathrm{Ce} 23,3^{\circ} \mathrm{C}$, respectivamente. O nível de $28 \mathrm{~m}$, por encontrar-se praticamente inserido no interior do dossel, foi o que apresentou os maiores valores médios de todas as alturas estudadas,

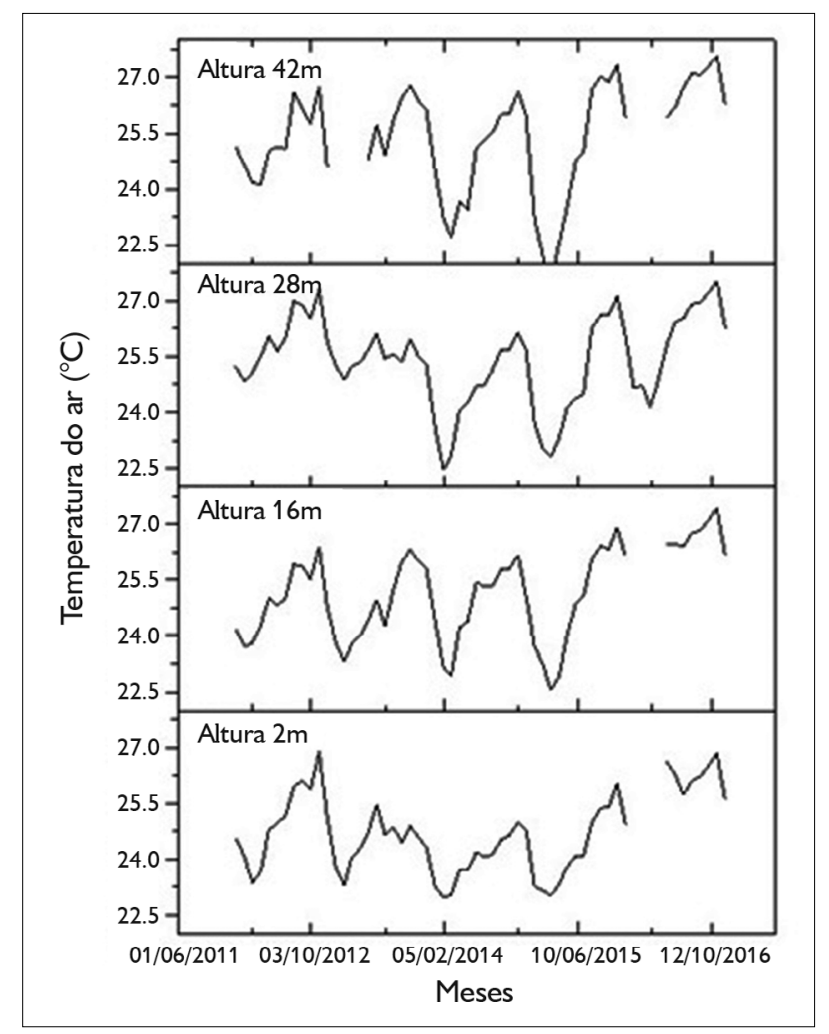

Figura 3. Variabilidade média anual do perfil de temperatura do ar no sítio experimental do projeto ESECAFLOR/LBA, no período de 2012 a 2016.

com $25,4^{\circ} \mathrm{C}$, superando, inclusive, o nível do topo, acima do dossel. Os máximos valores médios $\left(26,7^{\circ} \mathrm{C}\right)$ se aproximaram bastante daqueles observados acima do dossel $\left(26,9^{\circ} \mathrm{C}\right)$, sendo este nível caracterizado como um dos mais aquecidos entre todos os estudados. Acima do dossel (42 m), os valores médios foram de $25,1^{\circ} \mathrm{C}$, entretanto, foi também onde ocorreram os menores valores de todas as alturas estudadas $\left(22,9^{\circ} \mathrm{C}\right)$, certamente associado ao intenso resfriamento radiativo noturno, em função da maior velocidade do vento neste nível, o qual foi também onde se observaram as maiores amplitudes térmicas diárias, com valores de $4,1^{\circ} \mathrm{C}$. No nível da superfície (2 m), pela total ausência de ventos e, consequentemente, pela fraca mistura do ar, as amplitudes térmicas foram as menores observadas no perfil estudado, com apenas $2,5^{\circ} \mathrm{C}$. 


\section{VARIABILIDADE MÉDIA MENSAL DO PERFIL DE TEMPERATURA DO AR}

Na Figura 4, temos a variabilidade média mensal do perfil de temperatura do ar no sítio experimental do projeto ESECAFLOR/LBA, no período de 2012 a 2016. Durante os anos estudados, o padrão de variação do perfil vertical da temperatura do ar não apresentou alterações consideráveis, tendo as alturas de $2 \mathrm{~m}$ e de $16 \mathrm{~m}$ apresentado os menores valores médios de temperatura do perfil estudado, sendo estes valores médios mensais de $24,7^{\circ} \mathrm{C}$ e de $25,1^{\circ} \mathrm{C}$, respectivamente. As alturas de $28 \mathrm{~m}$ e de $42 \mathrm{~m}$ foram as mais quentes do perfil, ambas com valores médios mensais de $25,4^{\circ} \mathrm{C}$. As maiores diferenças foram encontradas nas amplitudes térmicas mensais durante o período estudado, tendo

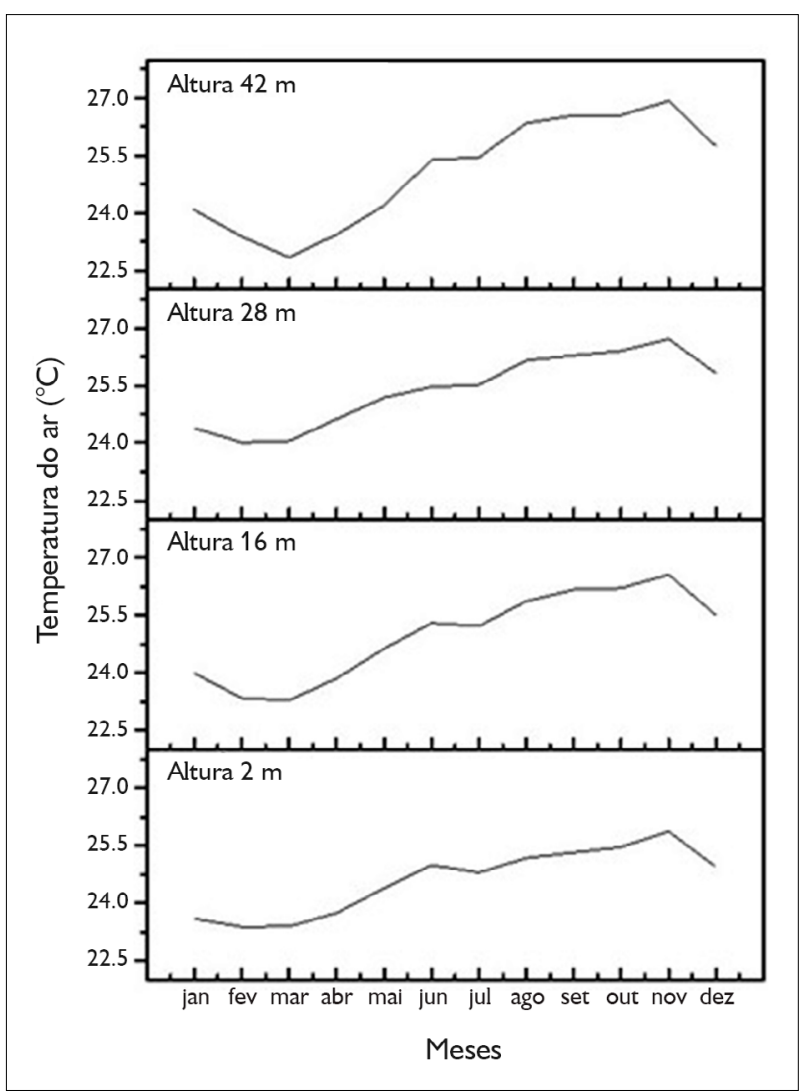

Figura 4. Variabilidade média mensal do perfil de temperatura do ar no sítio experimental do projeto ESECAFLOR/LBA, no período de 2012 a 2016. estas aumentado progressivamente dos menores para os maiores níveis de altura, sendo estas amplitudes de 3,9 ${ }^{\circ} \mathrm{C}(2 \mathrm{~m}), 4,9^{\circ} \mathrm{C}(16 \mathrm{~m}), 5,1^{\circ} \mathrm{C}(28 \mathrm{~m})$ e $6,2^{\circ} \mathrm{C}(42 \mathrm{~m})$. Esta característica deve estar relacionada basicamente com a atenuação da radiação solar global pelo dossel vegetativo e com o perfil vertical do vento neste ecossistema florestal, sendo zero próximo à superfície, aumentando logaritmicamente com a altura.

\section{VARIABILIDADE MÉDIA HORÁRIA DO PERFIL DE TEMPERATURA DO AR}

No estudo da variabilidade média horária do perfil de temperatura e de umidade relativa do ar, foram adotados quatro períodos distintos, chamados de madrugada ( 0 horas e 30 minutos a 6 horas), manhã (6 horas e 30 minutos a 12 horas), tarde (12 horas e 30 minutos a 18 horas) e, finalmente, noite ( 18 horas e 30 minutos a 24 horas). Na Figura 5, temos a variabilidade média horária do perfil de temperatura do ar durante o período chuvoso da região no sítio experimental do projeto ESECAFLOR/LBA, no período de 2012 a 2016. Observou-se que, de uma maneira geral, as menores temperaturas ocorreram no horário da madrugada, para todos os níveis, ao passo que as maiores foram observadas no horário da tarde. Em ambos os horários, o nível de $28 \mathrm{~m}$ foi o que apresentou os maiores valores, com médias de $22,7^{\circ} \mathrm{C}$ (madrugada) e $27,8^{\circ} \mathrm{C}$ (tarde).

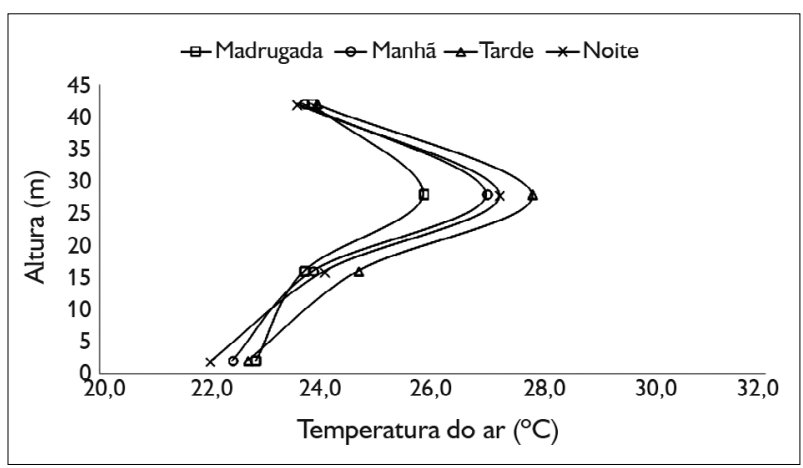

Figura 5. Variabilidade horária do perfil de temperatura do ar durante o período chuvoso da região no sítio experimental do projeto ESECAFLOR/LBA, entre 2012 a 2016.

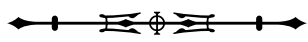


Os valores mais próximos foram observados entre os horários da noite e da madrugada, sendo que nos dois níveis inferiores apresentaram diferenças de $1,0^{\circ} \mathrm{C}(2 \mathrm{~m})$ e de $1,3{ }^{\circ} \mathrm{C}(16 \mathrm{~m})$, sofrendo uma pequena diferença nos níveis superiores (28 m e $42 \mathrm{~m}$ ).

Na Figura 6, temos a variabilidade média horária do perfil de temperatura do ar durante o período seco da região no sítio experimental do projeto ESECAFLOR/LBA, no período de 2012 a 2016. Observou-se, neste caso, que o padrão da variação vertical sofreu grande diferença em relação ao observado durante o período chuvoso, sendo que estes valores foram consideravelmente maiores em todos os níveis. As menores temperaturas ocorreram no horário da madrugada, para todos os níveis, ao passo que as maiores foram observadas no horário da tarde. Nos horários da manhã, da tarde e da noite, o nível de $28 \mathrm{~m}$ foi o que apresentou os maiores valores, com médias de $24,7^{\circ} \mathrm{C}$ (manhã), $27,8^{\circ} \mathrm{C}$ (tarde) e de $23,9^{\circ} \mathrm{C}$ (noite), e durante a madrugada o maior valor registrado foi de $22,7^{\circ} \mathrm{C}$ a $2 \mathrm{~m}$. Os valores foram muito próximos em todos os horários nos dois níveis inferiores (2 m e $16 \mathrm{~m}$ ), com médias variando entre $22,8^{\circ} \mathrm{C}(2 \mathrm{~m})$ a $27^{\circ} \mathrm{C}(16 \mathrm{~m})$, respectivamente.

\section{VARIABILIDADE MÉDIA HORÁRIA DO PERFIL DE UMIDADE RELATIVA DO AR}

A Figura 7 mostra a variabilidade média horária do perfil de umidade relativa do ar durante o período chuvoso da região no sítio experimental do projeto ESECAFLOR/ LBA, no período de 2012 a 2016. Como a umidade relativa do ar varia inversamente com a temperatura do ar, observou-se que, de uma maneira geral, os maiores valores de umidade relativa do ar ocorreram justamente nos horários onde foram observadas as menores temperaturas, ou seja, no período da madrugada, para todos os níveis, ao passo que os menores valores foram observados nos horários da tarde e da noite.

A Figura 8 ilustra a variabilidade média horária do perfil da umidade relativa do ar durante o período seco da

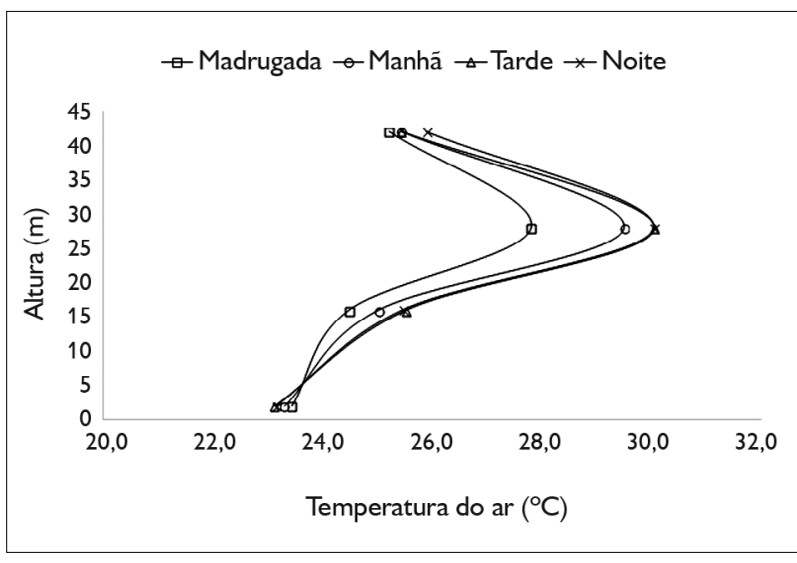

Figura 6. Variabilidade horária do perfil de temperatura do ar durante o período seco da região no sítio experimental do projeto ESECAFLOR/LBA, entre 2012 a 2016.

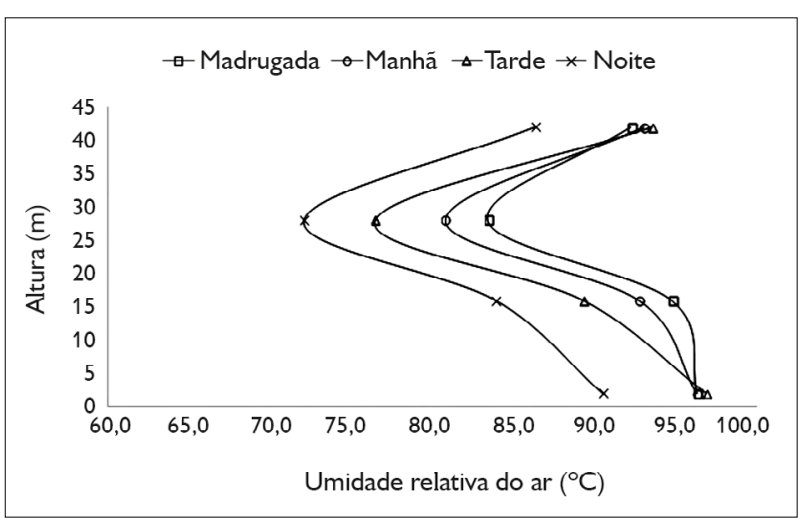

Figura 7. Variabilidade horária do perfil de umidade relativa do ar durante o período chuvoso da região no sítio experimental do projeto ESECAFLOR/LBA, entre 2012 a 2016.

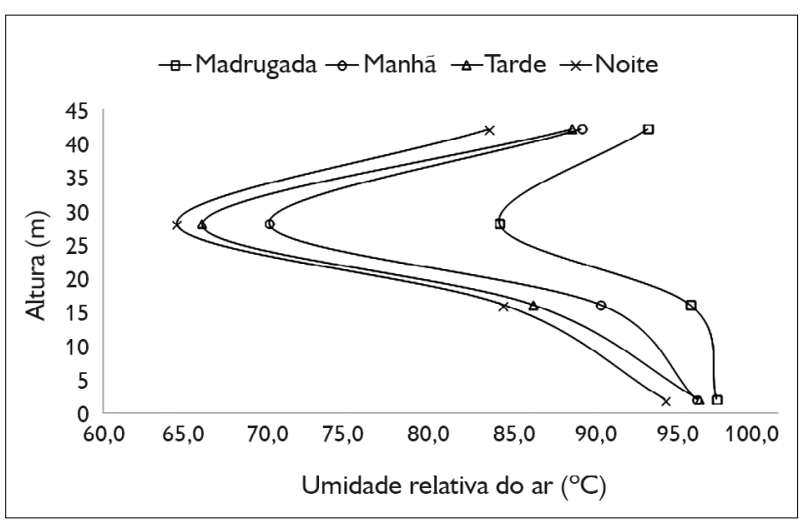

Figura 8. Variabilidade horária do perfil de umidade relativa do ar durante o período seco da região no sítio experimental do projeto ESECAFLOR/LBA, entre 2012 a 2016.

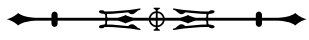


região no sítio experimental. Observou-se que, embora o padrão da variação vertical tenha se mantido próximo ao presenciado durante o período chuvoso, estes valores foram consideravelmente menores e mais próximos entre si em todos os níveis. Assim como no período chuvoso, neste período seco também se observou que os valores mais próximos foram registrados entre os horários da tarde e da noite, sendo que nos dois níveis inferiores (2 e $16 \mathrm{~m}$ ) eles foram muito próximos, apresentando discreta diferença nos níveis superiores (28 e 42 m).

\section{CONCLUSÃO}

Após as análises realizadas, ficou evidente e bem definida a sazonalidade do perfil de temperatura e de umidade relativa do ar, sendo que os menores valores de temperatura do ar ocorreram durante a época chuvosa da região, acontecendo o inverso com a umidade relativa do ar.

Em termos de altura, os menores valores médios de temperatura do ar foram observados nos níveis mais próximos à superfície, e os maiores aconteceram nos mais elevados.

Em termos de horários, os menores valores médios de temperatura do ar foram observados nas primeiras horas do dia em todos os níveis, e os maiores aconteceram nos horários da tarde.

Em termos gerais, observaram-se grandes variações, tanto em relação aos horários quanto às alturas no interior da floresta, o que indica grande heterogeneidade destes elementos meteorológicos no interior da floresta, podendo ter grande influência no padrão de distribuição da flora e da fauna nestes ambientes.

\section{AGRADECIMENTOS}

Os autores agradecem o apoio financeiro do Conselho Nacional de Desenvolvimento Científico e Tecnológico (CNPq), para a execução do projeto LBA/ESECAFLOR na Floresta Nacional de Caxiuanã, estado do Pará, e ao Museu Paraense Emílio Goeldi (MPEG).

\section{REFERÊNCIAS}

COSTA, A. C. L., A. P. BRAGA, R. F. COSTA, P. H. L. GONÇALVES, J. A. SILVA JUNIOR, P. MEIR \& Y. MALHI, 2004. Variações sazonais das temperaturas do ar e do solo sob influência de uma simulação de seca prolongada na Floresta de Caxiuanã. Anais do Congresso Brasileiro de Meteorologia 13: 1-8.

COSTA, A. C. L., D. GALBRAITH, S. S. ALMEIDA, B. T. T. PORTELA, M. COSTA, J. A. SILVA JUNIOR, A. P. BRAGA, P. H. L. GONÇALVES, A. A. R. OLIVEIRA, R. FISHER, O. L. PHILLIPS, D. B. METCALFE, P. LEVY \& P. MEIR, 2010. Effect of $7 \mathrm{yr}$ of experimental drought on vegetation dynamics and biomass storage of an eastern Amazonian rainforest. New Phytologist 187(3): 579-591. DOI: $<$ https://doi.org/10.1111/j.1469-8137.2010.03309.x>.

COSTA, R. F., V. P. R. SILVA, M. L. P. RUIVO, P. MEIR, A. C. L. COSTA, Y. S. MALHI, A. P. BRAGA, P. H. L. GONÇALVES, J. A. SILVA JUNIOR \& J. GRACE, 2007. Transpiração em espécie de grande porte na Floresta Nacional de Caxiuanã, Pará. Revista Brasileira de Engenharia Agrícola e Ambiental 11(2): 180-189. DOI: <http:// dx.doi.org/10.1590/S1415-43662007000200008>.

DANTAS, V. A., V. P. R. SILVA, A. C. L. COSTA \& G. F. B. CHAGAS, 2011. Fluxos de calor no dossel vegetativo e infiltração de água no solo, em floresta tropical. Revista Brasileira de Engenharia Agrícola e Ambiental 15(12): 1266-1274. DOI: < http://dx.doi.org/10.1590/ S1415-43662011001200008>.

FUNK, J. L. \& M. T. LERDAU, 2004. Photosynthesis in forest canopies. In: M. D. LOWMAN \& H. B. RINKER (Ed.): Forest canopies: 2. ed.: 335-358. Elsevier Academic Press, San Diego.

GEIGER, R., 1942. Das Klima der bodennahen Luftschicht: 2. ed.: 1-435. Vieweg, Braunschweig.

HOREL, J., A. HAHMANN \& J. GEISLER, 1989. An investigation of the annual cycle of the convective activity over the tropical Americas. Journal of Climate 2: 1388-1403. DOI: <https://doi. org/10.1175/1520-0442(1989)002<1388:AIOTAC > 2.0.CO;2>.

LISBOA, P. L. B., A. S. L. SILVA \& S. S. ALMEIDA, 1997. Florística e estrutura dos ambientes. In: P. L. B. LISBOA (Org.): Caxiuanã: 163-193. Museu Paraense Emílio Goeldi, Belém.

MADIGOSKY, S. R., 2004. Tropical microclimatic considerations. In: M. D. LOWMAN \& H. B. RINKER (Ed.): Forest canopies: 2. ed.: 24-48. Elsevier Academic Press, San Diego.

MALHI, Y., A. D. NOBRE, J. GRACE, B. KRUIJT, M. G. P. PEREIRA, A. CULF \& S. SCOTT, 1998. Carbon dioxide transfer over a Central Amazonian rain forest. Journal of Geophysical Research-Atmospheres 103(D24): 31593-31612. DOI: <https:// doi.org/10.1029/98JD02647>.

MALHI, Y., J. T. ROBERTS, R. A. BETTS, T. J. KILLEEN, W. LI \& C. A. NOBRE, 2008. Climate change, deforestation, and the fate of the amazon. Science 319(5860): 169- 172. DOI: <https://doi. org/10.1126/science.1146961>.

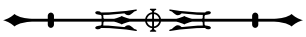


MEDEIROS, S. S., R. A. CECÍlIO, J. C. F. MELO JUNIOR \& J. L. C. SILVAJUNIOR, 2005. Estimativa e espacialização das temperaturas do ar mínimas, médias e máximas na região Nordeste do Brasil. Revista Brasileira de Engenharia Agrícola e Ambiental, 9(2): 247-255. DOI: <http://dx.doi.org/10.1590/S1415-43662005000200016>.

NEPSTAD, D. C., P. MOUTINHO, M. B. DIAS, E. DAVIDSON, G. CARDINOT, D. MARKEWITZ, R. FIGUEIREDO, N. VIANNA, J. CHAMBERS, D. RAY, J. B. GUERREIROS, P. LEFEBVRE, L. STERNBERG, M. MOREIRA, L. BARROS, F. Y. ISHIDA, I. TOHLVER, E. BELK, K. KALIF \& K. SCHWALBE, The effects of partial throughfall exclusion on canopy processes, aboveground production, and biogeochemistry of an Amazon forest. Journal of Geophysical Research 107(D20): LBA 53-1-LBA 53-18. DOI: https://doi. org/10.1029/2001JD000360.

NICOLASA, E., A. TORRECILLASA, M. F. ORTUNO, R. DOMINGO \& J. J. ALARCÓN, 2005. Evaluation of transpiration in adult apricot trees from sap flow measurements. Agricultural Water Management 72(2): 131-145. DOI: <https://doi.org/10.1016/j. agwat.2004.09.008>.
RUIVO, M. L. P., S. B. PEREIRA, E. P. C. BUSSETI, R. F. COSTA, B. QUANZ, T. Y. NAGAISHI, P. J. OLIVEIRA, P. MEIR, Y. MALHI \& A. C. L. COSTA, 2002. Propriedades do solo e fluxo de $\mathrm{CO}_{2}$ em Caxiuanã, Pará: experimento LBA - ESECAFLOR. In: E. L. KLEIN, M. L. VASQUEZ \& M. L. ROSA-COSTA (Org.): Contribuições à Geologia da Amazônia: v. 3: 291-299. SBG-Núcleo Norte, Belém.

WORLD METEOROLOGICAL ORGANIZATION (WMO), 2008. Guide to meteorological instruments and methods of observation: 7. ed: 1-681. World Meteorological Organization (WMO, n. 8), Geneva. 
